MKG-Chirurg 2012 · 5:6-6

DOI 10.1007/s12285-011-0262-y

Online publiziert: 12. Februar 2012

๑) Springer-Verlag 2012

H.-F. Zeilhofer

Klinik für Mund-, Kiefer- und Gesichtschirurgie, Universitätsspital Basel

\title{
Gesicht und Identität
}

befürchtet mancher Patient den Verlust seiner personalen Integrität, also seiner Identität, die ihm durch den unverwechselbaren persönlichen und individuellen Gesichtsausdruck gegeben wird.

Was macht diese Identität aus, die uns überall begegnet und von der wir doch so wenig wissen? Ein Paläoanthropologe, der zu seinem Fach über die Zahnmedizin gefunden hat, ein Psychiater und eine MKGChirurgin, in Zusammenarbeit mit einem Informatiker, betrachten in den Leitartikeln das Thema „Gesicht und Identität“ jeweils aus ihrem individuellen Blickwinkel. Bei der Lektüre spüren wir, dass uns das Thema bis zum Äußersten fordert. Das Gesicht als Ausdruck der Identität wirft multidisziplinäre Fragestellungen auf. Wie werden Gesichter wahrgenommen? Welchen evolutionären Veränderungen verdankt der Mensch die Fähigkeit zur Gesichtserkennung? Wie weit lassen sich - vor allem durch chirurgische Prozesse - Ästhetik und Ausdrucksfähigkeit von Gesichtern beeinflussen? Woher beziehen wir überhaupt unsere ästhetischen Leitbilder für die Attraktivität eines Gesichts, und wo liegen die Grenzen für verändernde Maßnahmen und Gesichtsrekonstruktionen? Die chirurgische Ausbildung allein befähigt uns nicht, geeignete Antworten zu geben. Wir benötigen Unterstützung aus vielen Fachgebieten, nicht nur der Medizin oder der Psychologie, sondern auch der Philosophie, der Theologie, der Soziologie, den Naturwissenschaften und auch der Kunst. erhalten oder wieder zurückzugeben. Die ästhetische Gesichtschirurgie ist zu einer wichtigen, unverzichtbaren Teildisziplin unseres Fachs geworden. Täglich erleben wir, dass Patienten, die sich einer Gesichtsoperation unterziehen, spezielle Ängste äußern oder besondere ästhetische Anforderungen an ihren Arzt des Vertrauens adressieren. Anders als bei einer Bauchoperation
Eine erfolgreiche wissenschaftliche Beschäftigung mit dem komplexen Thema erfordert zwingend den interdisziplinären Approach. Welche Antworten die aktuelle Forschung geben kann, wird vom 14. bis 16. Juni 2012 an der Universität Basel beim Internationalen Bernd-Spiessl-Symposium diskutiert, wenn sich dort zum achten Mal in ununterbrochener Reihenfolge Mediziner, Mathematiker, Natur- und Geisteswissenschaftler, Ingenieure und Künstler mit „Gesicht und Identität“ befassen. In der MKG-Chirurgie kommt uns zu Hilfe, dass das Fach an sich schon genuin interdisziplinär angelegt ist: Medizin und Zahnmedizin, zu einer neuen Einheit verschmolzen, strahlen auf weitere Disziplinen aus. Ich bin persönlich fest davon überzeugt, dass in der Zukunft „Gesicht und Identität“ in den Katalog der Fort- und Weiterbildung Eingang finden wird wie die Ethik oder die Implantologie. Es ist kein Zufall, dass mit Prof. Bernard Devauchelle ausgerechnet ein MKGChirurg die erste Gesichtstransplantation durchführte und dadurch das menschliche Antlitz weltweit zu einem öffentlichen Thema machte.

Ich hoffe, die Leser dieser Ausgabe finden in den Beiträgen zum Thema "Gesicht und Identität“ eine Vielzahl von Anregungen für ihre tägliche Arbeit.

\section{lans. Forth bille Yry}

\section{H.-F. Zeilhofer}

\section{Korrespondenzadresse}

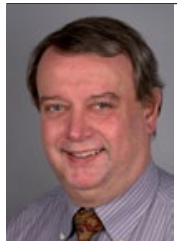

Prof. Dr. Dr. Dr. h. c. H.-F. Zeilhofer Klinik für Mund-, Kiefer- und Gesichtschirurgie, Universitätsspital Basel Spitalstr. 21, 4031 Basel Schweiz hfzeilhofer@uhbs.ch 